\title{
Observation and Characterization of Fragile Organometallic Molecules Encapsulated in Single-Wall Carbon Nanotubes
}

\author{
Daisuke Ogawa, ${ }^{1,2}$ Ryo Kitaura, ${ }^{1}$ Takeshi Saito, ${ }^{3}$ Shinobu Aoyagi, ${ }^{4}$ Eiji Nishibori, ${ }^{5}$ \\ Makoto Sakata, ${ }^{6}$ Tetsuya Nakamura, ${ }^{6}$ and Hisanori Shinohara ${ }^{1}$ \\ ${ }^{1}$ Department of Chemistry, Nagoya University, Nagoya 464-8602, Japan \\ ${ }^{2}$ Department of Chemistry, The University of Tokyo, Tokyo 113-0033, Japan \\ ${ }^{3}$ Nanotube Research Center, National Institute of Advanced Industrial Science and Technology, Tsukuba 305-8565, Japan \\ ${ }^{4}$ Department of Information and Biological Science, Nagoya City University, Nagoya 467-8501, Japan \\ ${ }^{5}$ Division of Physics, Faculty of Pure and Applied Sciences, University of Tsukuba, 1-1-1 Tennodai, Tsukuba, Ibaraki 305-8571, Japan \\ ${ }^{6}$ Japan Synchrotron Radiation Research Institute, Hyogo 679-5198, Japan
}

Correspondence should be addressed to Hisanori Shinohara; noris@nagoya-u.jp

Received 26 February 2014; Accepted 21 April 2014; Published 9 June 2014

Academic Editor: Keita Kobayashi

Copyright (C) 2014 Daisuke Ogawa et al. This is an open access article distributed under the Creative Commons Attribution License, which permits unrestricted use, distribution, and reproduction in any medium, provided the original work is properly cited.

\begin{abstract}
Thermally fragile tris $\left(\eta^{5}\right.$-cyclopentadienyl)erbium $\left(\mathrm{ErCp}_{3}\right)$ molecules are encapsulated in single-wall carbon nanotubes (SWCNTs) with high yield. We realized the encapsulation of $\mathrm{ErCp}_{3}$ with high filling ratio by using high quality SWCNTs at an optimized temperature under higher vacuum. Structure determination based on high-resolution transmission electron microscope observations together with the image simulations reveals the presence of almost free rotation of each ErCp $\mathrm{p}_{3}$ molecule in SWCNTs. The encapsulation is also confirmed by X-ray diffraction. Trivalent character of Er ions (i.e., Er ${ }^{3+}$ ) is confirmed by X-ray absorption spectrum.
\end{abstract}

\section{Introduction}

Nanowires with a few atoms in diameter show completely different properties from those of the three-dimensional bulk materials [1-11]. However, in general, those ultimately thin nanowires are unstable under atmospheric conditions and they are therefore quite difficult to realize.

In 1998, it was found that single-wall carbon nanotubes (SWCNTs) are able to encapsulate $\mathrm{C}_{60}$ fullerenes in their inner hollow space [12]. Since the discovery, various materials have been encapsulated in SWCNTs, which has produced a wide variety of low-dimensional hybrid nanomaterials; SWCNTs have provided ideal nanospace and protective walls for encapsulated materials [13-17]. In addition to their specific structure, these newly formed hybrid nanomaterials have shown interesting properties [18-20].

The encapsulation of organometallic complex molecules, such as $\mathrm{Co}\left(\mathrm{C}_{5} \mathrm{H}_{5}\right)_{2}$ [21] and $\mathrm{Fe}\left(\mathrm{C}_{5} \mathrm{H}_{5}\right)_{2}$ [22-25], in the
SWCNTs has attracted wide attention due to an expected control of SWCNT's electronic properties through charge transfer between encapsulates and SWCNT. Furthermore, organometallics can act as a precursor to form metal atomic wires in SWCNTs via the so-called nanotemplate reaction, which may lead to the formation of novel metal atomic wires.

Encapsulation of organometallics in SWCNTs, however, has so far been difficult, mainly because of the fact that, under atmospheric condition or at high temperature, such materials normally decompose during the encapsulation process. Here, we have focused on the development of a versatile method to encapsulate fragile organometallic complexes in SWCNTs. To confirm the encapsulation, we have employed a structure determination procedure that is based on high-resolution transmission electron microscope (HR-TEM) observations and HR-TEM image simulation by the multislice method [26]. 


\section{Experimental}

SWCNTs were synthesized by the so-called extended direct injection pyrolytic synthesis (e-DIPS) [27]. As-produced e-DIPS SWCNTs (a-CNTs) were annealed at $1200^{\circ} \mathrm{C}$ in vacuum $\left(\sim 10^{-5} \mathrm{~Pa}\right)$ for 14 hours in order to remove remaining Fe catalyst nanoparticles and amorphous carbon impurities [28]. Before the encapsulation reaction, the purified e-DIPS SWCNTs (p-CNTs) were heated under dry air flow at $600^{\circ} \mathrm{C}$ for $30 \mathrm{~min}$ in order to open the endcap of SWCNTs.

$\mathrm{ErCp}_{3}$ is an air- and moisture-sensitive material and decomposes easily upon exposure to the atmosphere [29]. $\mathrm{ErCp}_{3}$ was thus carefully handled and purified by sublimation at $200^{\circ} \mathrm{C}$ under high vacuum $\left(\sim 10^{-4} \mathrm{~Pa}\right)$ [29] and was kept in an anaerobic glove box. In the following experiments, all the sample preparation was performed using the purified $\mathrm{ErCp}_{3}$ under anaerobic condition to avoid any undesired degradation of $\mathrm{ErCp}_{3}$ during the preparation. Open-ended p-CNTs (o-CNTs) were vacuum sealed $\left(\sim 10^{-4} \mathrm{~Pa}\right)$ in a Pyrex tube with the purified $\mathrm{ErCp}_{3}$, and the sealed Pyrex tube was heated at $250^{\circ} \mathrm{C}$ for 72 hours. At this temperature and pressure, $\mathrm{ErCp}_{3}$ was sublimed and encapsulated in the hollow space of the SWCNTs. The as-prepared ErCp $\mathrm{p}_{3} @$ o-CNTs were washed with anhydrous tetrahydrofuran in order to remove any $\mathrm{ErCp}_{3}$ molecules physically adsorbed on the outer surface of the SWCNTs. The final products were dried at $80^{\circ} \mathrm{C}$ for 12 hours. As a control experiment, we also prepared a sample, $\mathrm{ErCp}_{3} @ \mathrm{p}-\mathrm{CNTs}$, by performing the encapsulation process of $\mathrm{ErCp}_{3}$ on $\mathrm{p}-\mathrm{CNTs}$.

\section{Results and Discussion}

Figure 1 shows a thermal gravimetric analysis (TGA) trace of a-CNTs and p-CNTs. Two shoulders were clearly seen at around $400^{\circ} \mathrm{C}$ and $600^{\circ} \mathrm{C}$ on the TGA curve of a-CNTs. The former and the latter shoulders correspond to the oxidation of amorphous carbon and the oxidation of SWCNTs and graphite, respectively. In contrast, only one sharp drop at $500^{\circ} \mathrm{C}$ and a smaller amount of residual material (3.84 wt.\%) were observed in the TGA curve of p-CNTs, which indicates higher purity of $\mathrm{p}$-CNTs than that of a-CNTs.

Figures 2(a) and 2(b) are HR-TEM images of aCNTs and p-CNTs, respectively. As seen in Figure 2(a), dark contrasts of Fe catalyst nanoparticles and amorphous impurity attached outer surface of SWCNTs are clearly observed. The amount of $\mathrm{Fe}$ catalyst nanoparticles and amorphous impurity greatly decreases after purification, as shown in Figure 2(b). In Figures S1(a) and (b) (see Figures S1(a) and S1(b) in Supplementary Material available online at http://dx.doi.org/10.1155/2014/539295), the corresponding energy dispersive X-ray (EDX) spectra are shown. Obtained spectra clearly show that the amount of residual Fe had been greatly reduced by purification. These HR-TEM images are consistent with the TGA results.

Figures 3(a) and 3(b) show HR-TEM images of ErCp 3 @oCNTs having different tube diameters. As seen in Figure 3(a), dot-like contrasts align in one-dimensional fashion at intervals of $0.94 \mathrm{~nm}$ inside SWCNTs whose diameter is $1.30 \mathrm{~nm}$.

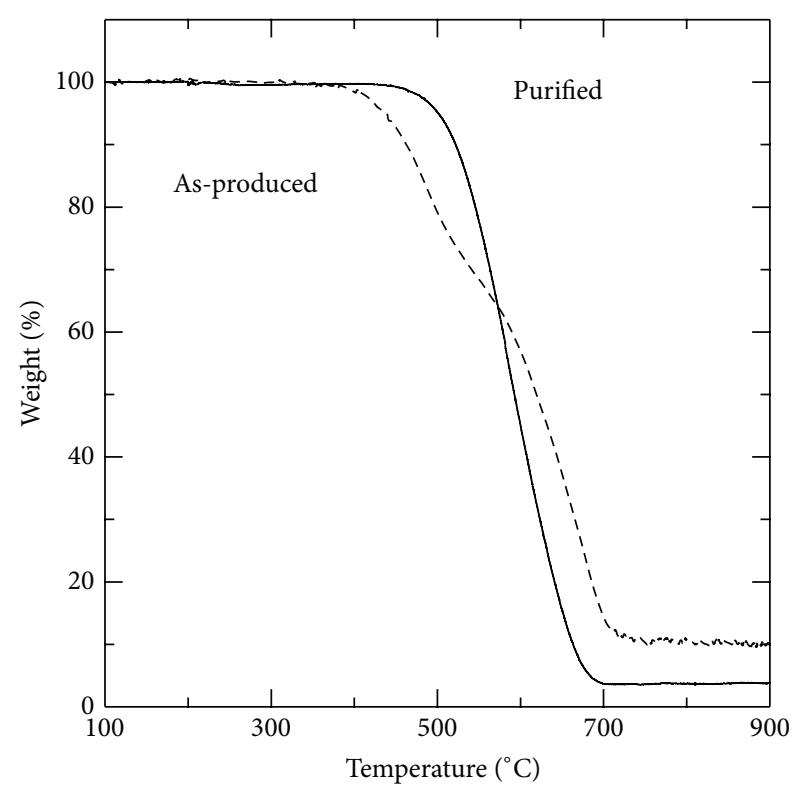

FIGURE 1: TGA profiles of as-produced and purified e-DIPS SWCNT. The sharp drop in sample weight at higher temperature and the smaller amount of residual material show higher quality of purified SWCNTs than that of as-produced ones.

The observed interval of $0.94 \mathrm{~nm}$ is almost the same with the molecular size of the $\mathrm{ErCp}_{3}$ molecule; the molecular size was estimated considering van der Waals radius of constituent atoms. Moreover, the difference between the diameter of SWCNTs (i.e., $1.30 \mathrm{~nm}$ ) and the intervals of dot-like contrasts (i.e., $0.94 \mathrm{~nm}$ ) observed is nearly equal to the van der Waals radii of the inner wall of SWCNTs $(\sim 0.34 \mathrm{~nm})[30,31]$.

When the diameter of SWCNTs is larger than $1.30 \mathrm{~nm}$, $\mathrm{ErCp}_{3}$ molecules aggregate to form clusters (Figure 3(b)). An EDX spectrum observed in the same area shows strong peaks that can be attributed to $\operatorname{Er} M_{\alpha}, L_{\alpha}$, and $L_{\beta}$ (Figure $\mathrm{S} 1(\mathrm{c})$ ). The dark spots were not observed in o-CNTs prior to the $\mathrm{ErCp}_{3}$ encapsulation, and no EDX peaks from $\mathrm{Er}$ atoms were observed. Therefore, we conclude that observed contrasts arise from encapsulated $\mathrm{ErCp}_{3}$ molecules forming one-dimensional regular array in hollow space of SWCNTs. The estimated filling ratio from the HR-TEM images is ca. $30 \%$ or more, which is substantially higher than that previously reported for organometallic complexes molecules encapsulated in SWCNTs [21, 22].

To confirm the high filling ratio of $\mathrm{ErCp}_{3}$ in o-CNTs, we have measured X-ray diffraction (XRD) patterns. Figures S2(a) and (b) show the XRD patterns of o-CNTs, ErCp $3 @$ oCNTs, p-CNTs, and ErCp $\mathrm{p}_{3} @ \mathrm{p}-\mathrm{CNTs}$. As shown in Figures S2(a) and (b), the number of diffraction peaks is limited, so that it is difficult to determine precise filling ratio by pattern fitting. Filling of ErCp $\mathrm{p}_{3}$ molecules, however, can be confirmed by changes in intensity of (10) peak. As clearly seen in Figure S2(a), the intensity of (10) peak of ErCp $\mathrm{F}_{3} @ \mathrm{o}-\mathrm{CNT}$ is much smaller than that of o-CNTs. This is a clear indication that the inner space of o-CNTs is filled with guest materials (i.e., $\left.\mathrm{ErCp}_{3}\right)[14,32,33]$. 


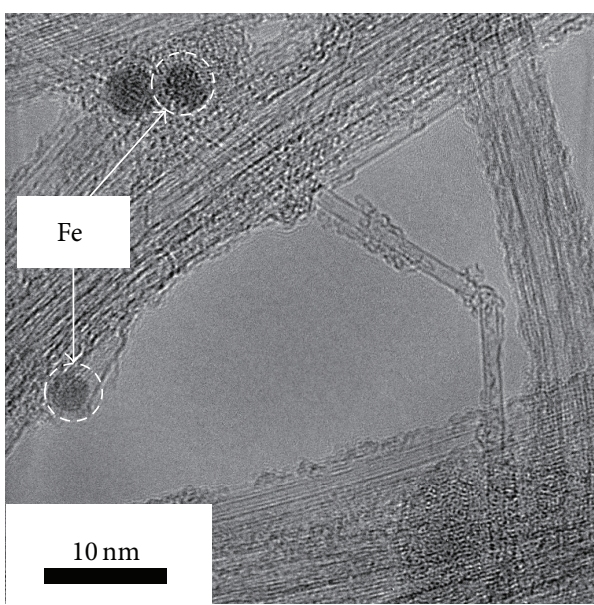

(a)

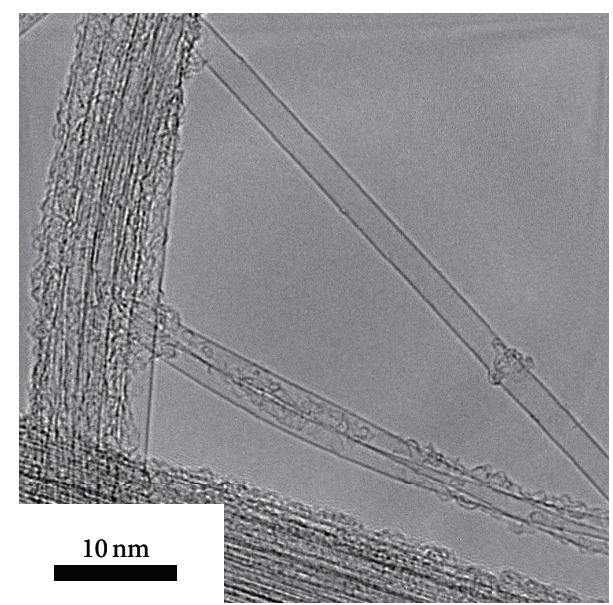

(b)

FIGURE 2: HR-TEM image of (a) as-produced and (b) purified e-DIPS SWCNTs.

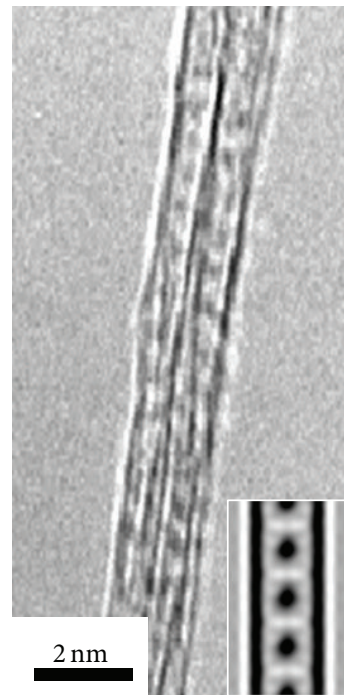

(a)

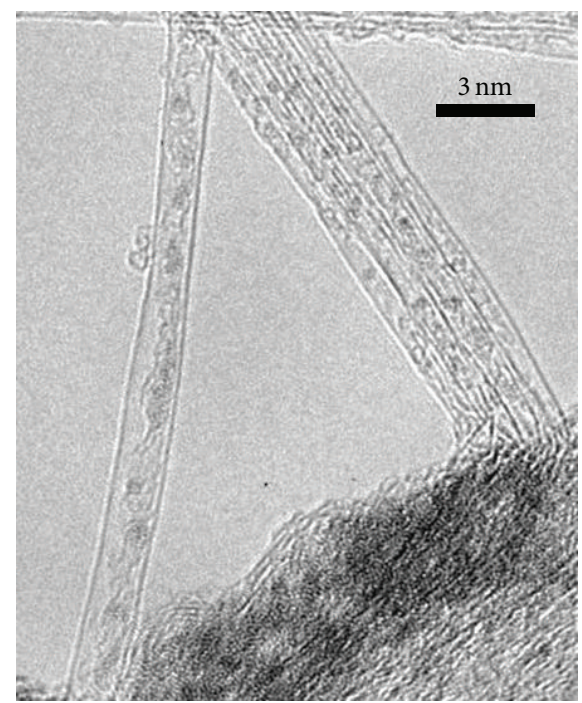

(b)

FIGURE 3: HR-TEM images of ErCp $p_{3}$ molecules in (a) thinner and (b) broader SWCNTs. The inset of (a) is a simulated HR-TEM image of $\mathrm{ErCp}_{3} @ \mathrm{o}-\mathrm{CNT}$.

The intensity of (10) peak of ErCp 3 @p-CNTs is comparable to that of p-CNTs, suggesting that the observed intensity drop in ErCp 3 @o-CNTs can be attributed to encapsulation of $\mathrm{ErCp}_{3}$ molecules (Figure S2(b)). The high filling ratio is caused by (1) purification and anaerobic handling of $\mathrm{ErCp}_{3}$, (2) an improved preparation method of o-CNTs (i.e., high quality, proper diameter, and optimized cap-opening conditions), and (3) performing the encapsulation reaction under high vacuum at optimized conditions.

Based on the observed HR-TEM images of $\mathrm{ErCp}_{3} @ S W C N T$, we have constructed a structure model. Image simulations by the multislice method (at a defocus of $600,650,700,750$, and $800 \mathrm{~nm}$ ) have been carried out based on the structure model constructed. To attain satisfactory agreements between the observed and simulated HR-TEM images, we have superimposed simulated HR-TEM images of $\mathrm{ErCp}_{3} @ S W C N T$ with different molecular orientations, in which simulated images based on fixed molecular orientations do not match with the observed images.

As illustrated in inset of Figure 3(a), the final simulated HR-TEM image well reproduces both the observed dark ellipsoids and their intensities. Hence, encapsulated $\mathrm{ErCp}_{3}$ molecules may be rotating much faster than the time scale of HR-TEM observation (the typical exposure time is several seconds). This suggests that the interaction between encapsulated $\mathrm{ErCp}_{3}$ and SWCNTs should fairly be weak. Since the ionization energy of $\mathrm{ErCp}_{3}(7 \sim 8 \mathrm{eV})$ is much larger than the threshold under which charge transfer interaction between SWCNTs occurs [14]; the interaction between $\mathrm{ErCp}_{3}$ and SWCNTs should not be significant. Here the ionization 


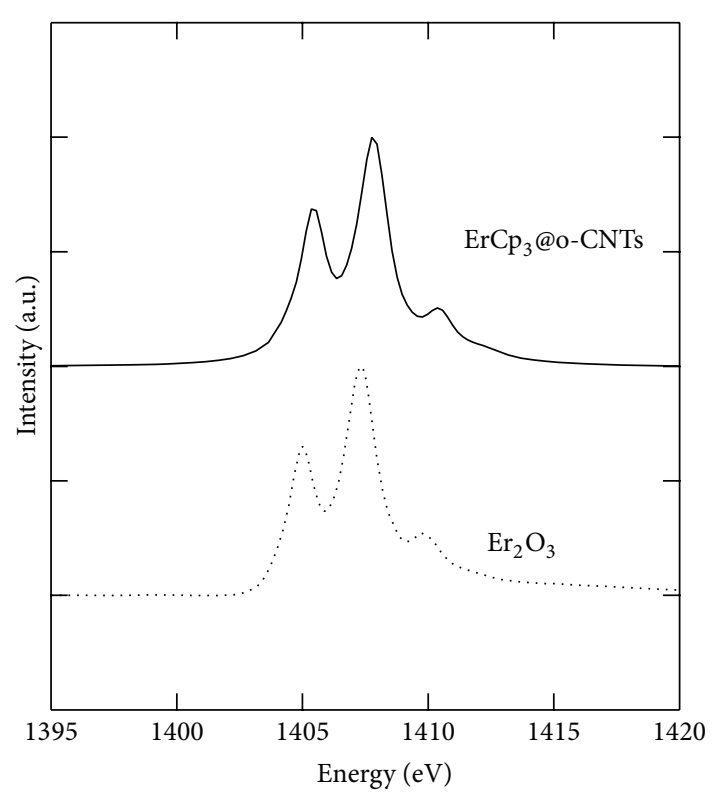

FIgURE 4: X-ray absorption spectra of ErCp $\mathrm{E}_{3} @ 0-\mathrm{CNTs}$ and $\mathrm{Er}_{2} \mathrm{O}_{3}$, confirming the presence of $\mathrm{Er}^{3+}$ in both species.

energy of $\mathrm{ErCp}_{3}$ was estimated from the ionization energy of other molecules, that is, $\mathrm{LaCp}_{3}(7.9 \mathrm{eV}), \operatorname{PrCp}_{3}(7.68 \mathrm{eV})$, and $\mathrm{TmCp}_{3}(7.43 \mathrm{eV})$, respectively [34].

To further investigate the electronic structure of $\mathrm{ErCp}_{3}$, $\mathrm{X}$-ray absorption spectrum (XAS) measurements at the Er $M_{5}$ absorption edge were performed, which indicates trivalency of Er ions (i.e., $\mathrm{Er}^{3+}$ ) (Figure 4). The spectrum is almost identical to that of $\mathrm{Er}_{2} \mathrm{O}_{3}$, suggesting that encapsulated $\mathrm{ErCp}_{3}$ molecules do not transform into any clusters or aggregates as such. SWCNTs act only as a template that restricts the space where $\mathrm{ErCp}_{3}$ molecules are encapsulated. The restricted space of SWCNTs is, therefore, well suited to stabilize unstable metal-containing complexes and to create low-dimensional alignment of various metal complexes including unstable organometallics.

\section{Conclusions}

We have successfully fabricated novel low-dimensional crystalline $\mathrm{ErCp}_{3}$ nanowires encapsulated in SWCNTs with filling yield of $\sim 30 \%$ and characterized their structural properties. Encapsulation reactions carried out under high temperature and high vacuum conditions using high quality SWCNTs are

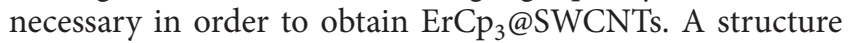
determination method based on the simulated annealing method and HR-TEM image simulation has been shown to be useful in characterizing the crystal structure of metal complex nanowires formed in SWCNTs. The present study may lead to future fabrication of various low-dimensional metal complexes in SWCNTs in high yield.

\section{Conflict of Interests}

The authors declare that there is no conflict of interests regarding the publication of this paper.

\section{Acknowledgments}

Hisanori Shinohara acknowledges the Grant-in-Aid for Scientific Research (S) (no. 22225001) of MEXT, Japan, for the partial support of the present study. The XRD experiments were performed at BL02B2 and XAS experiments were performed at BL25SU in SPring-8 with the approval of JASRT.

\section{References}

[1] Y. Wu, J. Xiang, C. Yang, W. Lu, and C. M. Lieber, "Singlecrystal metallic nanowires and metal/semiconductor nanowire heterostructures," Nature, vol. 430, no. 6995, pp. 61-65, 2004.

[2] Y. Kondo and K. Takayanagi, "Synthesis and characterization of helical multi-shell gold nanowires," Science, vol. 289, no. 5479, pp. 606-608, 2000.

[3] R. He and P. Yang, "Giant piezoresistance effect in silicon nanowires," Nature Nanotechnology, vol. 1, no. 1, pp. 42-46, 2006.

[4] J. Xiang, W. Lu, Y. Hu, Y. Wu, H. Yan, and C. M. Lieber, "Ge/Si nanowire heterostructures as high-performance fieldeffect transistors," Nature, vol. 441, no. 7092, pp. 489-493, 2006.

[5] B. R. McNamara, P. E. J. Nulsen, M. W. Wise et al., "The heating of gas in a galaxy cluster by X-ray cavities and large-scale shock fronts," Nature, vol. 433, no. 7021, pp. 45-47, 2005.

[6] T. Yoshie, A. Scherer, J. Hendrickson et al., "Vacuum Rabi splitting with a single quantum dot in a photonic crystal nanocavity," Nature, vol. 432, no. 7014, pp. 200-203, 2004.

[7] L. Mohaddes-Ardabili, H. Zheng, S. B. Ogale et al., "Selfassembled single-crystal ferromagnetic iron nanowires formed by decomposition," Nature Materials, vol. 3, no. 8, pp. 533-538, 2004.

[8] D. D. D. Ma, C. S. Lee, F. C. K. Au, S. Y. Tong, and S. T. Lee, "Small-diameter silicon nanowire surfaces," Science, vol. 299, no. 5614, pp. 1874-1877, 2003.

[9] B. H. Hong, S. C. Bae, C.-W. Lee, S. Jeong, and K. S. Kim, "Ultrathin single-crystalline silver nanowire arrays formed in an ambient solution phase," Science, vol. 294, no. 5541, pp. 348351, 2001.

[10] C. Zeng, P. R. C. Kent, T.-H. Kim, A.-P. Li, and H. H. Weitering, "Charge-order fluctuations in one-dimensional silicides," Nature Materials, vol. 7, no. 7, pp. 539-542, 2008.

[11] H. Ohnishi, Y. Kondo, and K. Takayanagi, "Quantized conductance through individual rows of suspended gold atoms," Nature, vol. 395, no. 6704, pp. 780-783, 1998.

[12] B. W. Smith, M. Monthioux, and D. E. Luzzi, "Encapsulated $\mathrm{C}_{60}$ in carbon nanotubes," Nature, vol. 396, no. 6709, pp. 323-324, 1998.

[13] J. Lee, H. Kim, S. J. Kahng et al., "Bandgap modulation of carbon nanotubes by encapsulated metallofullerenes," Nature, vol. 415, no. 6875 , pp. $1005-1008,2002$.

[14] T. Takenobu, T. Takano, M. Shiraishi et al., "Stable and controlled amphoteric doping by encapsulation of organic molecules inside carbon nanotubes," Nature Materials, vol. 2, no. 10, pp. 683-688, 2003.

[15] E. Philp, J. Sloan, A. I. Kirkland et al., "An encapsulated helical one-dimensional cobalt iodide nanostructure," Nature Materials, vol. 2, no. 12, pp. 788-791, 2003.

[16] M. A. Loi, J. Gao, F. Cordella et al., "Encapsulation of conjugated oligomers in single-walled carbon nanotubes: towards nanohybrids for photonic devices," Advanced Materials, vol. 22, no. 14, pp. 1635-1639, 2010. 
[17] T. Iwamoto, Y. Watanabe, T. Sadahiro, T. Haino, and S. Yamago, "Size-selective encapsulation of $\mathrm{C}_{60}$ by [10]cycloparaphenylene: formation of the shortest fullerene-peapod," Angewandte Chemie-International Edition, vol. 50, no. 36, pp. 8342-8344, 2011.

[18] J. Vavro, J. M. Kikkawa, and J. E. Fischer, "Metal-insulator transition in doped single-wall carbon nanotubes," Physical Review B, vol. 71, no. 15, Article ID 155410, 11 pages, 2005.

[19] A. Javey, R. Tu, D. B. Farmer, J. Guo, R. G. Gordon, and H. J. Dai, "High performance n-type carbon nanotube field-effect transistors with chemically doped contacts," Nano Letters, vol. 5, no. 2, pp. 345-348, 2005.

[20] E. Gaufres, N. Y.-W. Tang, F. Lapointe et al., "Giant Raman scattering from J-aggregated dyes inside carbon nanotubes for multispectral imaging," Nature Photonics, vol. 8, no. 1, pp. 72-78, 2013.

[21] L. J. Li, A. N. Khlobystov, J. G. Wiltshire, G. A. D. Briggs, and R. J. Nicholas, "Diameter-selective encapsulation of metallocenes in single-walled carbon nanotubes," Nature Materials, vol. 4, no. 6, pp. 481-485, 2005.

[22] Y. F. Li, R. Hatakeyama, T. Kaneko, and T. Okada, "Nano sized magnetic particles with diameters less than $1 \mathrm{~nm}$ encapsulated in single-walled carbon nanotubes," Japanese Journal of Applied Physics, vol. 45, no. 12-16, pp. L428-L431, 2006.

[23] Y. F. Li, T. Kaneko, T. Ogawa, M. Takahashi, and R. Hatakeyama, "Novel properties of single-walled carbon nanotubes with encapsulated magnetic atoms," Japanese Journal of Applied Physics, vol. 47, no. 4, pp. 2048-2055, 2008.

[24] H. Shiozawa, T. Pichler, R. Pfeiffer, H. Kuzmany, and H. Kataura, "Ferrocene encapsulated in single-wall carbon nanotubes: a precursor to secondary tubes," Physica Status Solidi B, vol. 244, no. 11, pp. 4102-4105, 2007.

[25] M. Sauer, H. Shiozawa, P. Ayala et al., "Environmental stability of ferrocene filled in purely metallic single-walled carbon nanotubes," Physica Status Solidi B, vol. 250, no. 12, pp. 25992604, 2013.

[26] S. Kirkpatrick, C. D. Gelatt Jr., and M. P. Vecchi, "Optimization by simulated annealing," Science, vol. 220, no. 4598, pp. 671-680, 1983.

[27] T. Saito, W. C. Xu, S. Ohshima, H. Ago, M. Yumura, and S. Iijima, "Supramolecular catalysts for the gas-phase synthesis of single-walled carbon nanotubes," Journal of Physical Chemistry $B$, vol. 110, no. 12, pp. 5849-5853, 2006.

[28] R. Kitaura, D. Ogawa, K. Kobayashi et al., "High yield synthesis and characterization of the structural and magnetic properties of crystalline $\mathrm{ErCl} 3$ nanowires in single-walled carbon nanotube templates," Nano Research, vol. 1, no. 2, pp. 152-157, 2008.

[29] J. M. Birmingham and G. Wilkinson, "The cyclopentadienides of scandium, yttrium and some rare earth elements," Journal of the American Chemical Society, vol. 78, no. 1, pp. 42-44, 1956.

[30] A. Bondi, "Van der Waals volumes and radii," Journal of Physical Chemistry, vol. 68, no. 3, pp. 441-451, 1964.

[31] S. Bandow, M. Takizawa, H. Kato, T. Okazaki, H. Shinohara, and S. Iijima, "Smallest limit of tube diameters for encasing of particular fullerenes determined by radial breathing mode Raman scattering," Chemical Physics Letters, vol. 347, no. 1-3, pp. 23-28, 2001.

[32] Y. Maniwa, Y. Kumazawa, Y. Saito et al., "Anomaly of X-ray diffraction profile in single-walled carbon nanotubes," Japanese Journal of Applied Physics, vol. 38, no. 6, pp. L668-L670, 1999.
[33] Y. Maniwa, H. Kataura, M. Abe et al., "Phase transition in confined water inside carbon nanotubes," Journal of the Physical Society of Japan, vol. 71, no. 12, pp. 2863-2866, 2002.

[34] S. G. Lias, J. E. Bartmess, J. F. Liebman, J. L. Holmes, R. D. Levin, and W. G. Mallard, Gas Phase Ion and Neutral Thermochemistry, Journal of Physical and Chemical Reference Data, vol. 17, Supplement, no. 1, The American Chemical Society and the American Institute of Physics for the National Bureau of Standards, New York, NY, USA, 1988. 

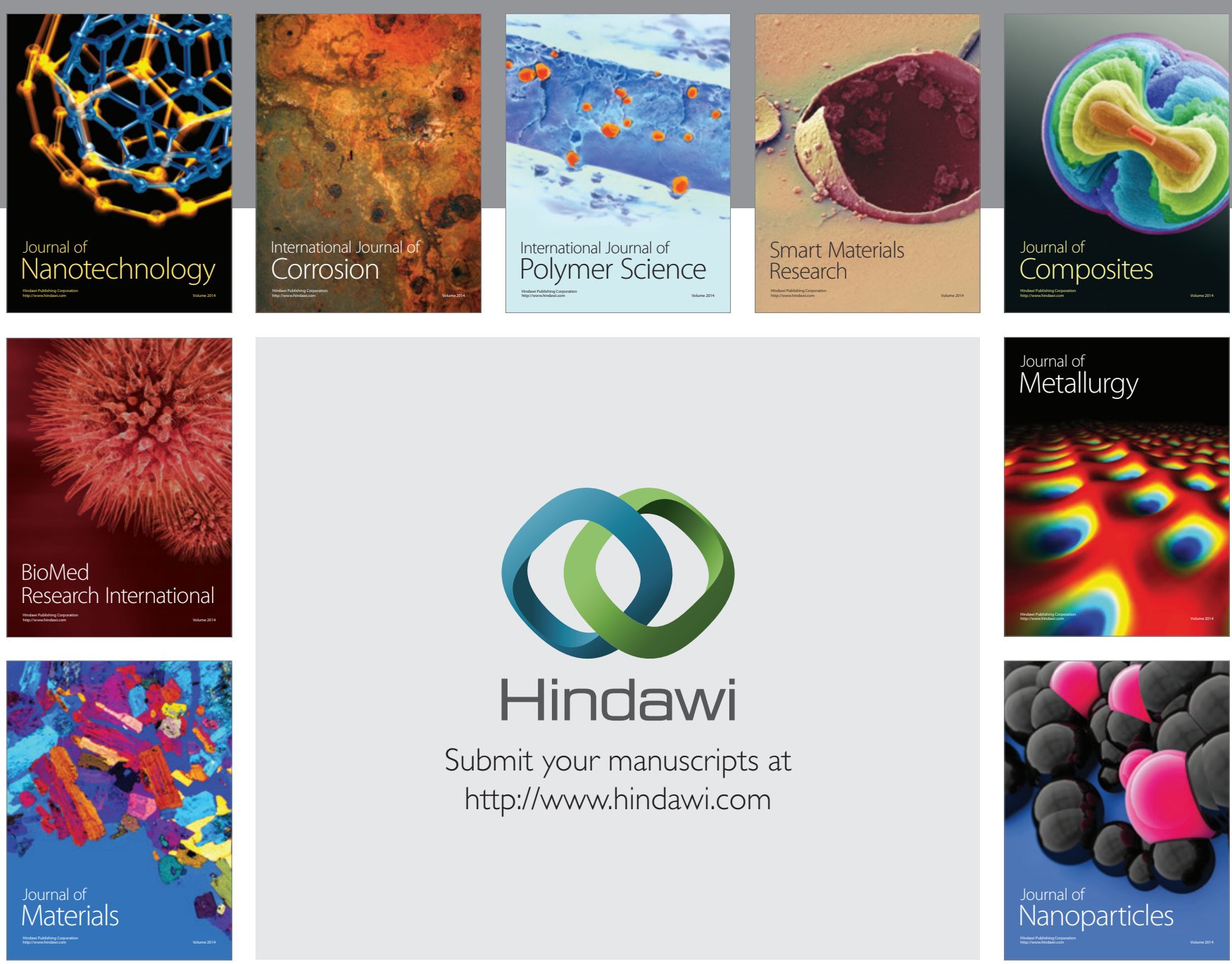

Submit your manuscripts at http://www.hindawi.com
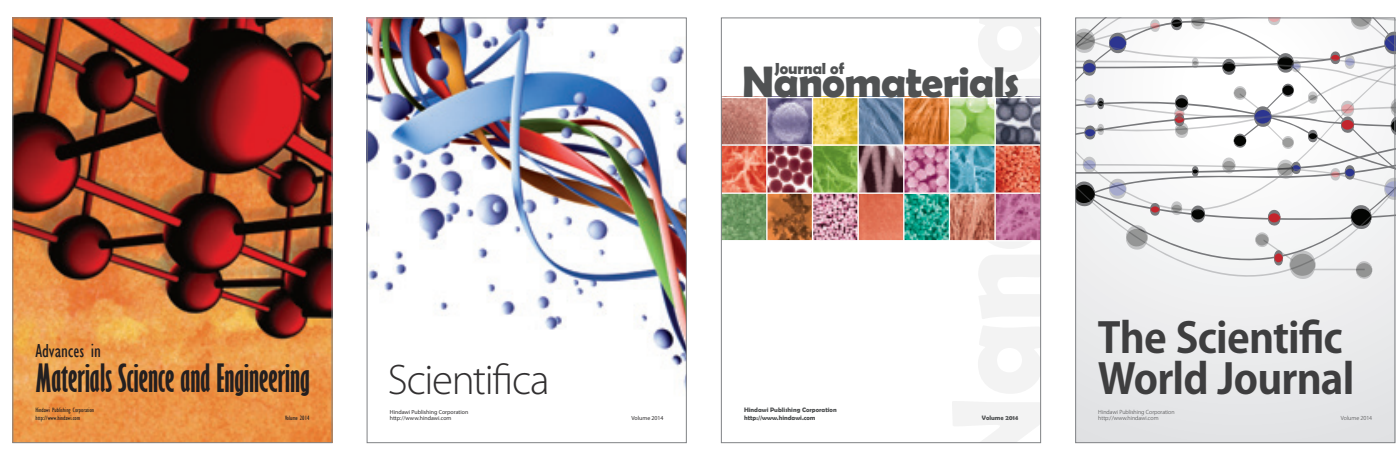

\section{The Scientific World Journal}
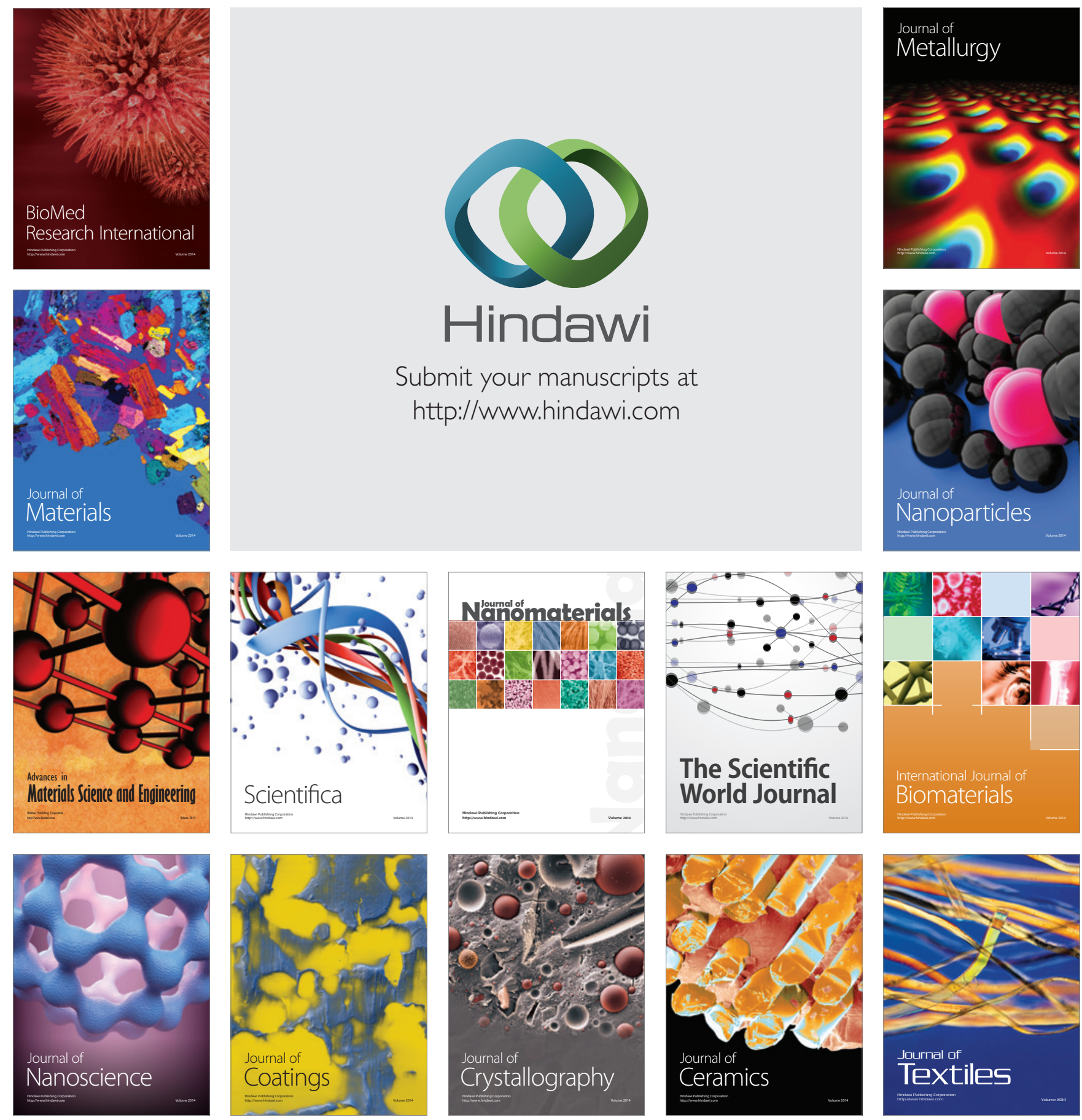